\title{
Laser-Fabricated Fluorescent, Ligand-Free Silicon Nanoparticles: Scale-up, Biosafety, and 3D Live Imaging of Zebrafish under Development
}

\author{
Marta d'Amora, ${ }^{\dagger, \ddagger}$ Marina Rodio, ${ }^{\dagger, \S, \perp}$ Giuseppe Sancataldo, ${ }^{\ddagger, \|}$ Alberto Diaspro, ${ }^{\ddagger}$ \\ and Romuald Intartaglia $* * \oplus$ \\ ${ }^{\ddagger}$ Nanophysics, Istituto Italiano di Tecnologia, Via Morego 30, Genoa 16163, Italy \\ ${ }^{\S}$ Hamburg Centre for Ultrafast Imaging, Luruper Chaussee 149, Hamburg 22761, Germany \\ ${ }^{\perp}$ Physical Chemistry, Hamburg University, Martin-Luther-King Platz 6, Hamburg 20146, Germany \\ "European Laboratory for Non-linear Spectroscopy (LENS), University of Florence, Sesto Fiorentino, Florence 50121, Italy
}

Supporting Information

\begin{abstract}
This work rationalizes the scalable synthesis of ultrasmall, ligand-free silicon nanomaterials via liquid-phase pulsed laser ablation process using picosecond pulses at ultraviolet wavelengths. Results showed that the irradiation time drives hydrodynamic NP size. Isolated, monodisperse Si-NPs are obtained at high yield (72\%) using post-treatment process. The obtained Si-NPs have an average size of $\sim 10 \mathrm{~nm}$ (not aggregated) and display photoemission in the green spectral range. We directly characterized the ligand-free Si-NPs in a vertebrate animal (zebrafish) and assessed their toxicity during the development. In vivo assay revealed that Si-NPs are found inside in all the early life stages of embryos and larvae growth, showing that the biosafety of Si-NPs and malformation types are independent of the Si-NP dose. Si-NPs were directly imaged inside developing embryos by spinning disk-imaging technique with optical sectioning capability. We showed that Si-NPs can passively enter inside embryos by the pore canals of chorion, can diffuse in the circulatory system, i.e., blood vessel, and accumulate inside larvae midgut and yolk sac, and in the eye lens, indicating the crossing of the blood barrier.
\end{abstract}

KEYWORDS: semiconductors, biocompatible materials, imaging agents, quantum dots, nanofabrication, laser ablation in liquid, biological materials, toxicology, translocation, blood barrier, biological imaging, fluorecence imaging, optical materials

\section{INTRODUCTION}

Semiconductor nanomaterials have gained attention for biomedical applications in imaging, drug delivery, and regenerative medecine. ${ }^{1,2}$ They can also potentially be used as multimodal agents in clinics, including photosensitizer, photoacoustic, ultrasound, and magnetic resonance imaging. ${ }^{3-7}$ However, a major concern limiting their medical applicability is the toxicity related to the traditional heavymetal nanoparticle, ${ }^{8}$ and the identity of the ligand itself, 9 i.e., surface chemistry. Silicon nanomaterials (Si-NPs) offer advantages of being composed elementally of nontoxic materials, biodegradable, essential human trace elements, and known to be metabolized by the body. ${ }^{10-14} \mathrm{Si}-\mathrm{NPs}$ possess strong absorption, size-tunable fluorescence emission, and good photostability, making them a promising nanoplatform for use as fluorescence imaging labeling, ${ }^{15-17}$ delivery vector, ${ }^{18-20}$ and theranostic agents for personalized biomedicine. $^{21,22}$ Previous works have shown that Si-NPs can be used in cancer applications, such as tumor targeting and lymph node biopsy, ${ }^{17}$ but also as self-tracking vehicles for delivering interfering RNA (siRNA) into tumor cells ${ }^{18}$ and quercetin anticancer drug. ${ }^{19}$

Whereas chemical routes ${ }^{23}$ prevail in Si-NP synthesis methods, others high-throughput and low-cost techniques including biomimetic green strategy $^{24}$ and physical approaches ${ }^{11,20}$ offer practical alternatives. In particular, liquid phase pulsed laser ablation (LP-PLA) permits the production of nanomaterials ${ }^{25-28}$ whose surface is free of any contamination, under biomolecule-friendly conditions. ${ }^{29,30}$ Besides that, LP-PLA approaches enable in one step the surface modification with molecules such as DNA, ${ }^{29}$ protein, ${ }^{15,30}$ and amino-silane. ${ }^{25}$ Briefly, a laser beam is focused onto the surface of a bulk material placed in aqueous medium. The nanomaterials growth mechanisms include nuclei, phase and kinetic transformations. ${ }^{31}$ The morphology of the laser-synthesized silicon-based nanomaterials can be adjusted by changing the initial concentration, ${ }^{21,32,33}$ the laser power, ${ }^{34,35}$ the irradiation

Received: October 11, 2018

Accepted: December 5, 2018

Published: December 5, 2018 
time, ${ }^{36,37}$ the laser wavelength, ${ }^{36,38}$ the pulse duration, ${ }^{39}$ or post-treatment. ${ }^{40}$ Bagga et al. reported Si-NPs-antibody nanoconjugates prepared by LP-PLA that can be used as universal secondary biomarkers for in vitro bioapplications. ${ }^{15}$ It has been demonstrated that Si-NPs prepared by LP-PLA can act as radiofrequency hyperthermia ${ }^{21}$ and photodynamic agents $^{22}$ for cancer therapy. Uncoated $40 \mathrm{~nm}$ Si-NPs prepared by LP-PLA are more efficient as RF-radiation sensitizers than those prepared by conventional method. ${ }^{21}$

Exposure to silicon-based nanomaterials in living tissues and organism is usually performed by administration of Si colloidal suspension. Several studies of silica nanomaterials, also known as silicon dioxide, have shown strong influences of size, shape, and concentration on the toxic behavior. ${ }^{41}$ Only few reports concerned the in vitro ${ }^{17,42,43}$ and in vivo ${ }^{44,45}$ Si-NPs studies, including toxicity, morphological and functional criteria. Studies found that the toxicity depends on the NPs size and surface chemistry and can lead to mitochondrial disruption and reactive oxygen species (ROS) production. ${ }^{42}$ Even if Si-NPs displays promising toxicity profiles, studies in more complex biological systems such as zebrafish (Danio Rerio) are essential to confirm toxicity concerns. ${ }^{46-48}$ Zebrafish possess several peculiarities in comparison to other vertebrate species (e.g., mouse). Embryos develop quickly, within $72 \mathrm{~h}$ post fertilization (hpf), with well-characterized developmental stages. It presents a high homology in physiology and molecular mechanisms to those of higher vertebrates, including humans. ${ }^{49}$ So far, exposure to silica nanomaterials in zebrafish has been discussed. ${ }^{50,51}$ Duan et al. showed that $60 \mathrm{~nm}$ silica nanoparticles in zebrafish induce high mortality, different types of malformations, and reduced locomotor activity. ${ }^{50}$ Others works revealed that dye-functionalized silica nanoparticles (>50 nm, green and red dye) do not internalize in zebrafish, and mostly accumulated on the chorion surface. ${ }^{51}$ Recently, it has been reported that nanomaterials surface have a role in NP toxicity, i.e., the ligand identity itself can influence toxicity. ${ }^{9}$ Even minimal ligand such as poly(allylamine hydrochloride) are demonstrated to cause significant toxicity when functionalized to nanoparticle. ${ }^{9}$ Thus, suitable NPs, i.e., free of toxic chemicals at the surface, are requested for translation into clinical setting.

Here, we report on the fabrication and scale-up protocol of ligand-free Si-NPs $(\sim 10 \mathrm{~nm})$ with photoemissive properties fabricated by LP-LPA, for studying their biodistribution imaging and long-term effects, i.e., toxicity, in early development zebrafish. Exposure to Si-NPs in zebrafish during the development are investigated in terms of survival and hatching rates, heart beat rate, locomotor activity, and potential presence of malformations. Our results indicate that Si-NPs have negligible toxicity to the zebrafish embryos at the concentrations tested $(5-100 \mathrm{ppm})$. Further, the in vivo biodistribution and functionality of Si-NPs are determined in zebrafish during the development by means of spinning diskimaging technique with optical sectioning capability.

\section{EXPERIMENTAL SECTION}

Fabrication and Characterization of Ligand-Free Si-NPs. SiNPs were prepared by ultraviolet picosecond laser ablation of silicon target in milli-Q water, as described previously. ${ }^{36}$ Briefly, a Si material bulk (Alpha Aesar, 99.999\%, Radius 6 mm, Height $10 \mathrm{~mm}$ ), was put on the bottom of a glass cuvette filled with $2 \mathrm{~mL}$ of deionized water. Laser beam from Nd:YAG picosecond laser (Continuum Leopard system, $355 \mathrm{~nm}, 20 \mathrm{ps}, 120 \mathrm{~mJ}, 20 \mathrm{~Hz}$ ) was focused with the use of a
$300 \mathrm{~mm}$ lens below the target surface $(10 \mathrm{~mm})$. Laser fluence of $3.5 \mathrm{~J}$ $\mathrm{cm}^{-2}$ was chosen to optimize the generation of NPs. ${ }^{36}$ The target was rotated at a velocity of $1 \mathrm{~mm} \mathrm{~s}^{-1}$ for uniform ablation. The thickness of the liquid layer above the Si bulk was around $13 \mathrm{~mm}$.

Size separation of the as-synthesized Si-NPs solution was achieved by ultracentrifugation (OPTIMA centrifuge with a TLA-55 Fixed Angle Rotor). In detail, the as-synthesized Si-NPs solution $(1000 \mu \mathrm{L})$ was centrifuged by tuning the parameters (rpm, $10000-40000$; time, 10-60 min). As result of the centrifugation, large Si-NPs deposit on the bottom of the cuvette (pellet, $100 \mu \mathrm{L}$ ), whereas the solution containing ultrasmall Si-NPs (supernatant, $900 \mu \mathrm{L}$ ) was separated and transferred into a blank glass vessel. Before analysis, supernatant and pellet were finally taken up in $1000 \mu \mathrm{L}$ of $\mathrm{dH} 2 \mathrm{O}$.

The concentration of Si-NPs, i.e., as-synthesized, supernatant, and pellet, was evaluated using ICP 6300 optical emission spectrometer (Thermos scientific). For that, Si-NPs $(100 \mu \mathrm{L})$ was placed in Aqua Regia as described in detail in previous work. ${ }^{30}$ The values were expressed in $\mathrm{ppm}\left(\mu \mathrm{g} \mathrm{mL}^{-1}\right)$, with error significance less than $0.5 \%$. Hydrodynamic diameter and zeta potential analysis of the Si-NPs were analyzed by a Zetasizer Nano ZS (Malvern Instruments). Triplicate measurements of the samples was performed and analyzed using Smoluchowski model. ${ }^{30}$

The morphology of Si-NPs was investigated using a transmission electron microscope, TEM (100 kV JEOL Jem 1011). High-resolution transmission electron microscopy, HR-TEM (200 kV JEOL JEM 2200FS) analyses were done as described in detail in previous report. $^{30}$ TEM and HR-TEM samples were prepared by depositing the solution onto a 300 mesh copper grids. Optical properties, i.e., absorption and photoluminescence, were investigated using a spectrophotometer (Cary 6000) and spectrofluorometer (Fluoroma4, Jobin Yvon-Horiba). The chemistry of Si-NPs was investigated using a Fourier transform infrared spectrometer (FTIR, Bruker $80 \mathrm{~V}$ ). $\mathrm{X}$-ray photoelectron spectroscopy (XPS) analysis were investigated using an AXIS Ultra DLD spectrometer (Kratos, CasaXPS software, version 2.3.16). ${ }^{27}$

Zebrafish Maintenance. Wild type zebrafish were kept at standard laboratory conditions. ${ }^{52}$ Briefly, embryos were obtained by photoinduced spawning, collected at $4-5 \mathrm{hpf}$, and rinsed several times with embryo medium.

Biosafety Evaluation. Healthy eggs were selected at 4 hpf by using a stereomicroscope (Zeiss Microscopy, Discovery.V8,) and exposed to different Si solutions (5, 10, 25, 50, and $100 \mathrm{ppm}$ ) for 4$120 \mathrm{hpf}$ following previous protocol. ${ }^{52}$ The different $\mathrm{Si}$ concentrations were prepared by diluting an initial $100 \mathrm{ppm} \mathrm{Si}$ solution and controlled by ICP-OES technique. Survival, hatching and heart beat rates, malformations, and locomotor activities were analyzed using the stereomicroscope (CCD camera) at various points time. The locomotor activity was recorded in larvae at $96 \mathrm{hpf}$, as previously reported by Duan et al. ${ }^{50}$

Uptake and Imaging. After treatment with Si-NPs, zebrafish at 24,48 , and $72 \mathrm{hpf}$ incubated with 25,50 , and $100 \mathrm{ppm}$ of Si-NPs were rinsed three times and anesthetized with tricaine (Sigma). Zebrafish were put in a Petri filled with embryo medium. The imaging was performed with a spinning-disk confocal microscope (SDCM) endowed with a Yokogawa CSU-X1 confocal scanning unit (FITC filter) and an Andor iXon EMCC (Quantum efficiency >90\%). A Nikon CFI Plan Fluor 4X/0.2 air objective was used for fluorescence measurements. A 488 laser was used to excite the nanoparticles and the emitted light was acquired in the range of $520-600 \mathrm{~nm}$. The exposure time of all images was $100 \mathrm{~ms}$. This technique provided optical section capability, low photo damage and fast imaging speed, suitable for 3D imaging of zebrafish. A total of six samples for each concentration and developmental stages were observed and imaged. All zebrafish assays were carried out in compliance with the revised law 2010/63/EU.

Statistical Analysis. The values are reported as means \pm the standard deviation from three independent experiments, and the data were analyzed as previously reported. ${ }^{48}$ 


\section{RESULTS AND DISCUSSION}

Si-NPs suspensions were fabricated by laser ablation of Si bulk directly in milli-Q water using ps pulses at ultraviolet wavelength $(355 \mathrm{~nm})$. (Figure 1) The Si-NPs hydrodynamic
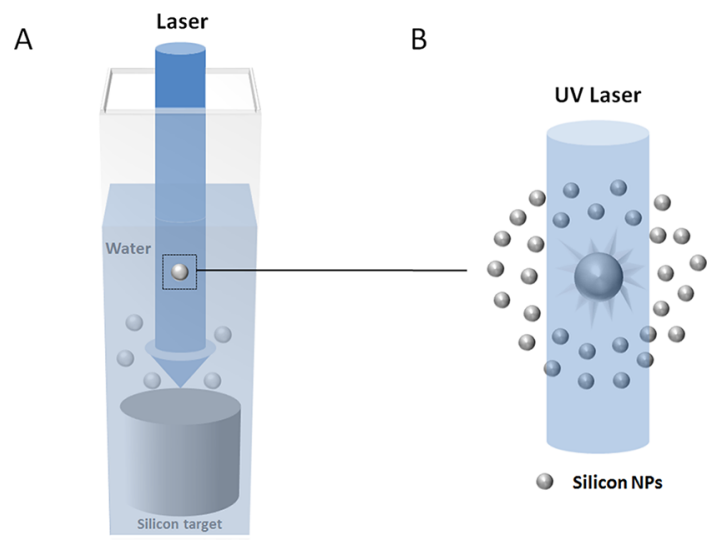

Figure 1. (A) Si-NPs suspensions fabricated by ps UV laser ablation of Si bulk in water. (B) Illustration of the in situ photofragmentation effect during laser ablation process.

diameter was monitored by the irradiation time adjustment. As demonstrated in Figure 2 by DLS analysis, Si-NPs suspensions

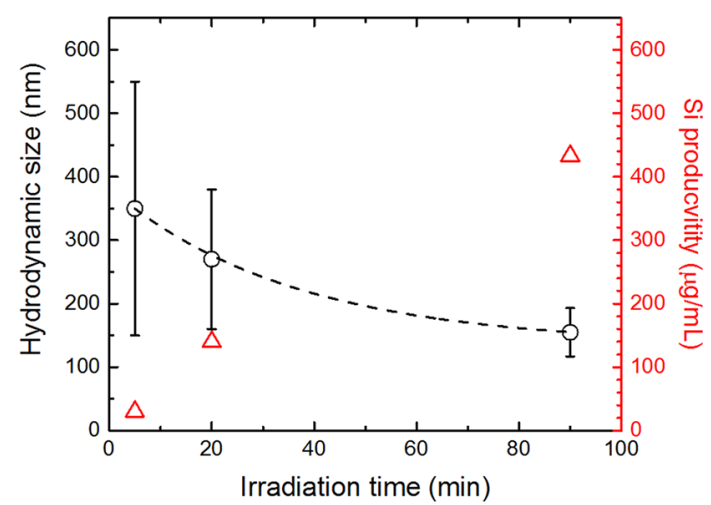

Figure 2. Hydrodynamic size (black circle) and Si productivity ( $\mathrm{Si}$ concentration, $\mu \mathrm{g} / \mathrm{mL}$, red triangle) as a function of irradiation time of Si colloidal solution prepared by UV LP-PLA at a fluence energy, $3.5 \mathrm{~J} \mathrm{~cm}^{-2}$. Error bar includes the $\mathrm{Si}$ colloids bimodal distribution (Figure S1B). Error bar is included in the symbol for Si productivity.

exhibited a hydrodynamic size of $(350 \pm 200) \mathrm{nm},(270 \pm$ $110) \mathrm{nm}$, and $(155 \pm 40) \mathrm{nm}$ at an irradiation time of 5,20 , and $90 \mathrm{~min}$, respectively. (Figure S1) These findings indicate that the UV laser can drive formation of nanoparticles and are associated with an in situ photofragmentation processes, i.e., the fragmentation of NPs into NPs of smaller size, by LP-PLA (Figure 1B). ${ }^{36}$ As to be demonstrated thereafter, the here large DLS values (Figure 2) resulted from the fact that the scattered light intensity from even a small number of residual large particles (i.e., not laser-fragmented) overshadows other coexisting particles, ultrasmall NPs. Of note, the mass productivity of Si-NPs increased non linearly with the irradiation time in accordance with the interplay between ablation and photofragmentation processes, ${ }^{36}$ reaching a value of $433 \mu \mathrm{g} \mathrm{mL}^{-1}$ at irradiation time of $90 \mathrm{~min}$. (Figure 2, Red triangle)
To guarantee effective uptake, uncoated Si-NPs size below $20 \mathrm{~nm}$ regime is ideal. ${ }^{53}$ The removal of the residual large SiNPs (i.e., not laser-fragmented) was evaluated by centrifugation protocols (rpm, time). (Figure $3 \mathrm{~A}, \mathrm{~B}$ and Figure S2) The

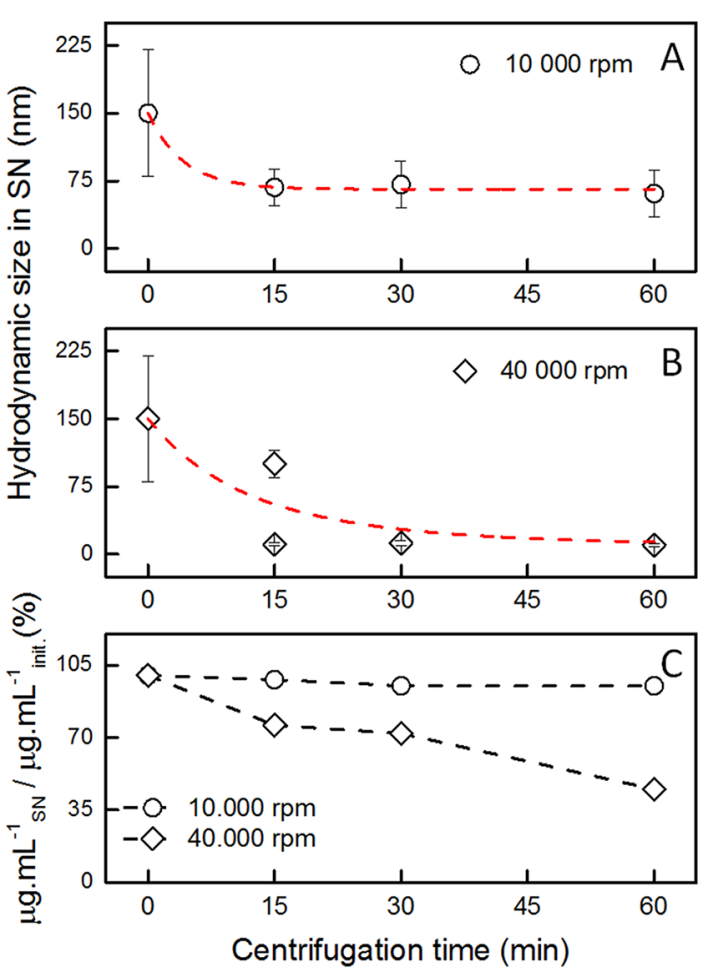

Figure 3. Hydrodynamic size of Si-NPs in the supernatant (SN) after centrifugation at (A) 10000 and (B) $40000 \mathrm{rpm}$ as a function of the time. At $40000 \mathrm{rpm}, 15 \mathrm{~min}$, two hydrodynamic sizes are observed, as shown by DLS analysis (Figure S2B). (C) Percentage ratio of Si concentration between the supernatant $\left(\mu \mathrm{g} \mathrm{mL}^{-1} \mathrm{SN}\right)$ and the initial solution $\left(\mu \mathrm{g} \mathrm{mL}_{\text {Init }}^{-1}\right)$ evaluated by ICP-EOS after centrifugation treatment (10000 rpm, $40000 \mathrm{rpm}$ ) for different centrifugation time.

process was completed after $30 \mathrm{~min}$ at $40000 \mathrm{rpm}$ leading to Si-NPs with a hydrodynamic diameter $(<10 \mathrm{~nm})$ (Figure 3B). Of note, the process is inoperative at 10000 r.p.m. even for long centrifugation times (Figure 3A). The Si concentration in the supernatant $\left(\mu \mathrm{g} \mathrm{mL}^{-1} \mathrm{SN}\right)$ with respect to the one in the initial solution $\left(\mu \mathrm{g} \mathrm{mL}^{-1}\right.$ Init), i.e., $433 \mu \mathrm{g} \mathrm{mL}^{-1}$ (Figure 2, $t=$ $90 \mathrm{~min}$ ), was evaluated by ICP-EOS analysis. Figure 3C shows that the percentage ratio $\left(\mu \mathrm{g} \cdot \mathrm{mL}^{-1}{ }_{\mathrm{SN}} / \mu \mathrm{g} \cdot \mathrm{mL}^{-1}{ }_{\text {Init }}\right)$ reaches a value around $72 \%$ and $45 \%$ after a centrifugation time of 30 and $60 \mathrm{~min}$, respectively. These results are supported by pellet ICP analysis (Figure S3). Optimal centrifugation parameters $(30 \mathrm{~min}, 40000 \mathrm{rpm}$ ) (Figure $3 \mathrm{~B}$ ) and ICP results (Figure 3C) yielded to Si-NPs (mean size $7 \mathrm{~nm}$ ) at a high yield, $312 \mu \mathrm{g}$ $\mathrm{mL}^{-1}$. The productivity efficiency of Si-NPs $(<10 \mathrm{~nm})$, i.e., $\mu \mathrm{g}$ $\mathrm{mL}^{-1}{ }_{\mathrm{SN}} / \mu \mathrm{g} \mathrm{mL}^{-1}{ }_{\text {Init }}$ after post-treatment, i.e., centrifugation, was evaluated at $\sim 72 \%$ (Figure $3 \mathrm{C}$ and Figure S3A).

$\mathrm{Si}$-NPs fabricated and post-treated were characterized for their physicochemical properties. As indicated in Figure 4A by DLS measurements, the post-treated Si-NPs exhibited a mean size of $(7 \pm 3) \mathrm{nm}$. Of note, larger cluster Si-NPs (i.e., not laser-fragmented) were efficiently removed in the centrifugation step as confirmed by DLS and absorption spectroscopy (Figures S4 and S5) The Si-NPs morphology under TEM analysis of post-treated Si-NPs confirmed monodispersed nanostructures (size $\approx 7 \mathrm{~nm}$ ) (Figure 5 and TEM images in 

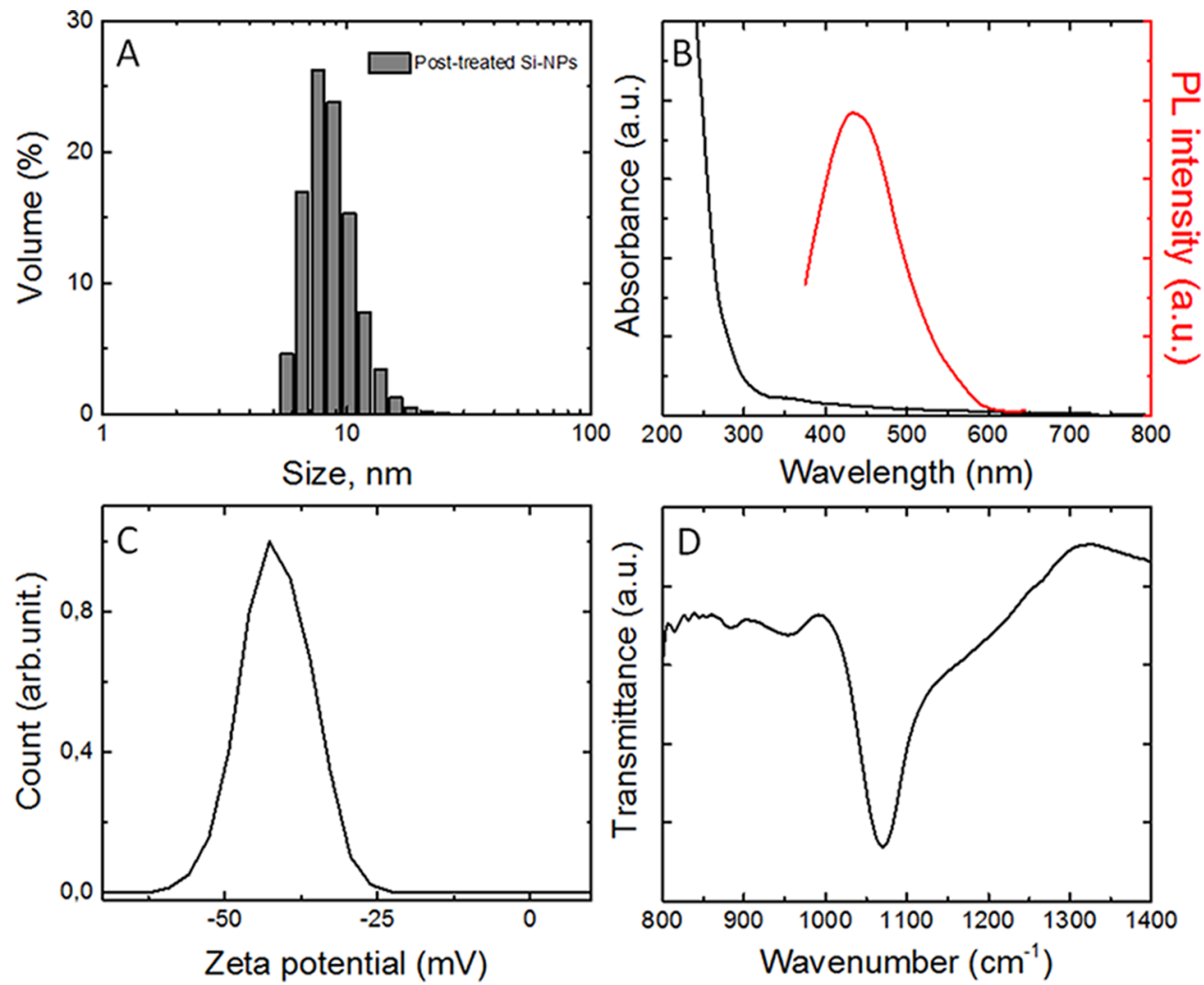

Figure 4. (A) Hydrodynamic diameter from DLS analysis and (B) Absorption and emission of post-treated Si-NPs obtained by UV LP-LPA, black and red line, respectively. (C) Surface zeta potential of Si-NPs colloidal solution by UV LP-PLA after post-treatment D) FTIR profile (800-1400 $\mathrm{cm}^{-1}$ ) of post-treated Si-NPs obtained by UV LP-LPA.

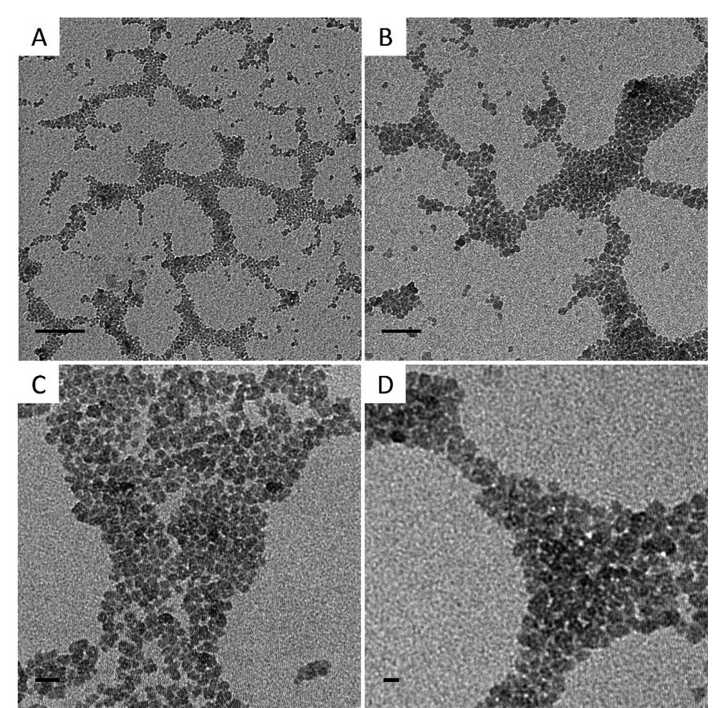

Figure 5. TEM observation of post-treated Si-NPs prepared by UV LP-PLA at various magnifications. Scale bars (A) 100, (B) 50, (C) 20, and (D) $10 \mathrm{~nm}$.

different grid areas in Figure S6). The surface zeta potential of Si-NPs $(<10 \mathrm{~nm})$ displayed a value of $(-41 \pm 4) \mathrm{mV}$ (Figure $4 C)$. The negative value results from the oxide-terminated group on NPs' surface, i.e., oxide layer less than $1 \mathrm{~nm}$ (Figure S7), which helps the stabilization of the Si-NPs. As to be explained thereafter, the water solubility of $\mathrm{Si}$ colloids results from the $\mathrm{Si}-\mathrm{O}-\mathrm{Si}$ coating the particles. ${ }^{25}$
Post-treated Si-NPs showed an absorbance starting ( 600 $\mathrm{nm}$ ) with a defined shoulder around $350 \mathrm{~nm}$ that is in accordance with the formation of ultrasmall Si-NPs. ${ }^{34,54}$ (Figure 4B, black line) Of note, before post-treatment, the absorption features of Si-NPs suspensions displayed a broad band in the visible region indicating the presence of large SiNPs (i.e., not fragmented). ${ }^{34}$ (Figure S4). Moreover, Si-NPs $(<10 \mathrm{~nm})$ emission revealed a PL feature at $450 \mathrm{~nm}$ (Figure $4 \mathrm{~B}$, red line), and at $515 \mathrm{~nm}$ (Figure S8) under excitation at 350 and $488 \mathrm{~nm}$, respectively. The Si-NPs emission feature is similar to those obtained previously using different multistep approaches. $^{54,55}$ The excitation wavelength dependent photoluminescence is in line with those of surface-oxidized Si-NPs features, ${ }^{54}$ which are attributed to the $\mathrm{SiO}_{x}$ defects states. ${ }^{56}$ Other reports attributed the PL origin to radiative centers from quantum confinement effects ${ }^{54}$ or from the oxide, i.e., $\mathrm{Si}-\mathrm{O}$ bonds, on the Si-NP surface. ${ }^{57}$

XPS analysis of Si-NPs indicated a $\mathrm{Si}-\mathrm{O}$ bonding surface coverage without other surface contamination. ${ }^{58,59}$ (Figure S9) FTIR studies confirmed the oxidized surface of Si-NPs with features that are similar to those obtained using different approaches. ${ }^{54,60,61}$ (Figure 4D) The main band $\left(1070 \mathrm{~cm}^{-1}\right)$ is due to $\mathrm{Si}-\mathrm{O}-\mathrm{Si}$ optical mode covering the particles. ${ }^{60}$ Numerous sharp peaks located at $800 \mathrm{~cm}^{-1}, 880$ and 960 $\mathrm{cm}^{-1}$ corresponding to various $\mathrm{SiOx}$ bending mode, ${ }^{61}$ nonbridging $\mathrm{Si}-\mathrm{O}$ bonds, $\mathrm{Si}-\mathrm{O}-\mathrm{Si}$ bonds, and $\mathrm{Si}-\mathrm{OH}$ bending $^{62}$ were observed. Moreover, transitions were seen around 1630, 2260, and in the range $3200-3650 \mathrm{~cm}^{-1}$, attributed to $\mathrm{SiO}-\mathrm{H}$ bending, ${ }^{63} \mathrm{OxSi}-\mathrm{H}$ vibrations modes, ${ }^{64}$ and the $\mathrm{O}-\mathrm{H}$ vibrational modes, ${ }^{65}$ respectively (Figure S10). 
Zebrafish Biological Screening. Exposure to Si-NPs $(\approx$ $10 \mathrm{~nm}$ ) in zebrafish was examined by treating embryos at different concentration of Si-NPs $(5,10,25,50$, and 100 ppm) (Experimental Section). The different toxicological end points were evaluated between 4 and 120 hpf (Figure S11). Figure 6 displays the survival and the hatching rates (chorion aperture).
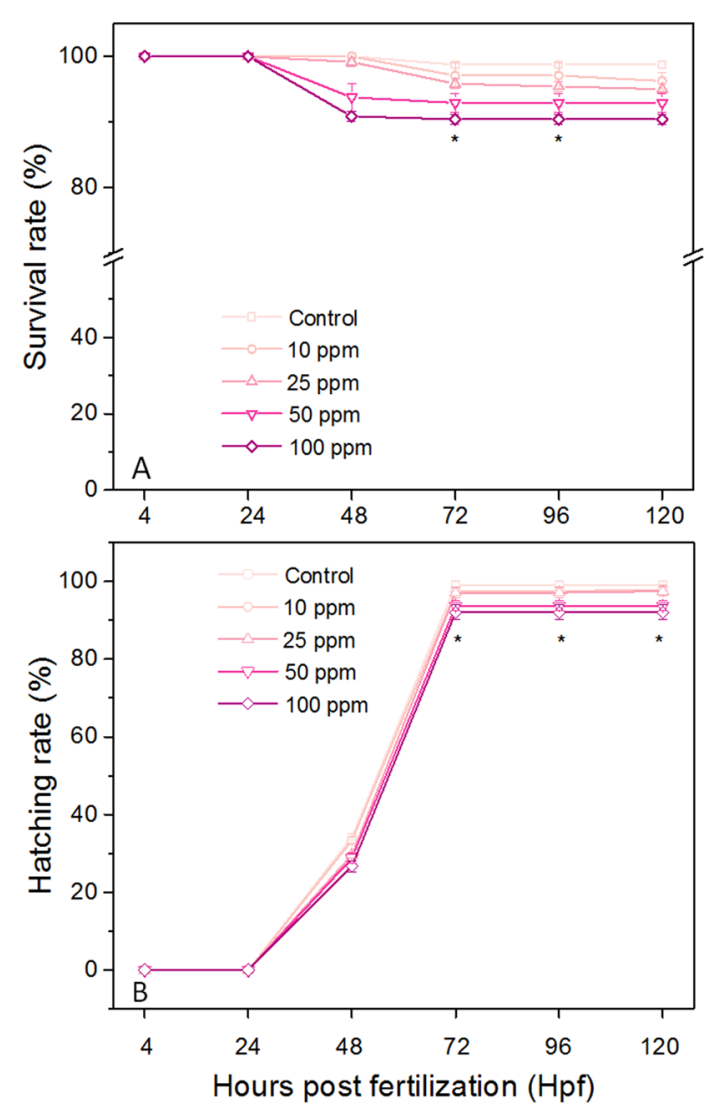

Figure 6. (A) Survival and (B) hatching rates of zebrafish after Si-NPs treatment at various concentration $(* p \leq 0.1$ in comparison to the control).

The survival rate showed a low decrease in a time and concentration manner, with a significant difference respect to the control, between 48 and $72 \mathrm{hpf}$ (Figure 6A). The chorion aperture presented a behavior time and concentration dependence, with no delay of the hatching rate of embryos (Figure 6B). The treated larvae hatched normally between 48 and $72 \mathrm{hpf}$. Of note, above $72 \mathrm{hpf}$ a significant difference ( $p \leq$ 0.1 ) at $100 \mathrm{ppm}$ was present in the hatching rate in comparison to the control. These values indicated nontoxic effects according to the normative law (OECD guidelines). These toxicological end points, i.e., survival and hatching rates, suggested the biosafety of ligand-free Si-NPs prepared by our protocol with respect to chemically synthesized polymer grafted Si-NPs. ${ }^{66}$

Furthermore, we examined the heart beat rate of zebrafish larvae treated with Si-NPs at $72 \mathrm{hpf}$. The average value of heartbeats decreased in a dose-dependent manner, with no relevant difference compared to the samples of control (Figure S12A). Also, the total swimming distance covered showed no reduction whether compared to controls (Figure S12B). Although silica NPs $(>50 \mathrm{~nm})$ presented significant bradycardia $^{50}$ and altered larval locomotor activity, ${ }^{50}$ our findings indicated that Si-NPs have no effect on the larval behavior. Others reports on nanomaterials being either nontoxic ${ }^{67}$ or toxic ${ }^{68}$ in nature induced a decrease in the heart beat number and swimming distance ${ }^{68}$ or affected swimming parameters (velocity and activity level). ${ }^{67}$

Abnormal activity induced by Si-NPs exposure was examined by embryos/larvae imaging test, at the late gastrula (12 hpf), hatching (48 hpf), and completed developed larvae stage (96 hpf). (Figure 7) Exposed embryos grew regularly
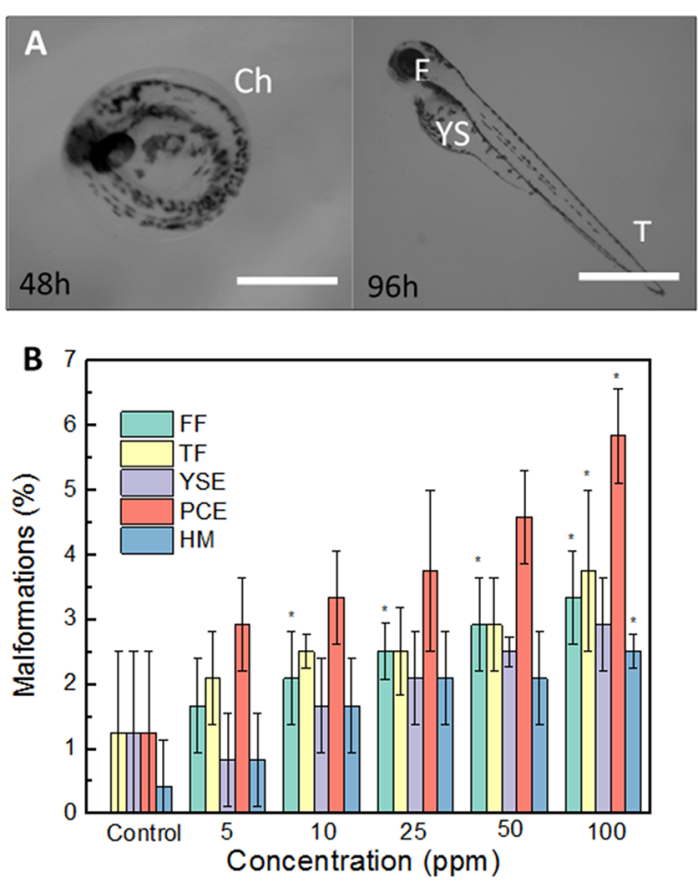

Figure 7. (A) Bright-field images of zebrafish at 48 and $96 \mathrm{hpf}$ treated with 100 ppm of Si-NPs. Scale bars $=500 \mu \mathrm{m}$. T, tail. Ch, chorion; E, eye; Ys, yolk sac. (B) Histograms of the percentage of abnormalities with each type of malformations versus Si-NPs concentrations at 96 hpf. HM, head malformations; PCE, pericardial edema; YSE, yolk sac edema; TF, tail flexure; and FF, fin fold flexure.

presenting a low malformation incidence. Si-NPs induced five different types developmental defects, including pericardial edema (PCE), head malformation (HM), finfold flexure (FF), and yolk sac edema (YSE) (Figure 7B). As Si-NPs concentration increased up to $100 \mathrm{ppm}$, the developmental defects percent reached $6 \%$ in the case of the pericardial edema (red column), indicating a low toxicity effect of the Si-NPs prepared by our protocol. Comparable effects were observed for polymer grafted Si-NPs, ${ }^{66}$ while others heavy-metal binary compound $^{68}$ displayed up to $60 \%$ of PCE.

Optical Sectioning and Si-NPs Localization. The SiNPs biodistribution in zebrafish was observed using SDCM microscopy with optical sectioning capability, at three different stages of zebrafish growth: 24, 48, and 72 hpf. Figures $8 \mathrm{~A}-\mathrm{C}$ show the maximum intensity projection of zebrafish embryos at $24 \mathrm{hpf}$ treated with 25,50 , and $100 \mathrm{ppm}$ of Si-NPs. Of note, the embryos at this stage ( $24 \mathrm{hpf})$ were surrounded until 48 hpf by the chorion, a protective barrier. We can observe that Si-NPs in part accumulate on the chorion surface of zebrafish embryos (green emission, Figure 8). As shown in Figure 8D, the intensity emission profile becomes higher in agreement with Si-NPs concentrations $(25,50,100 \mathrm{ppm})$. As to be discussed thereafter, the Si-NPs emission enables localization 

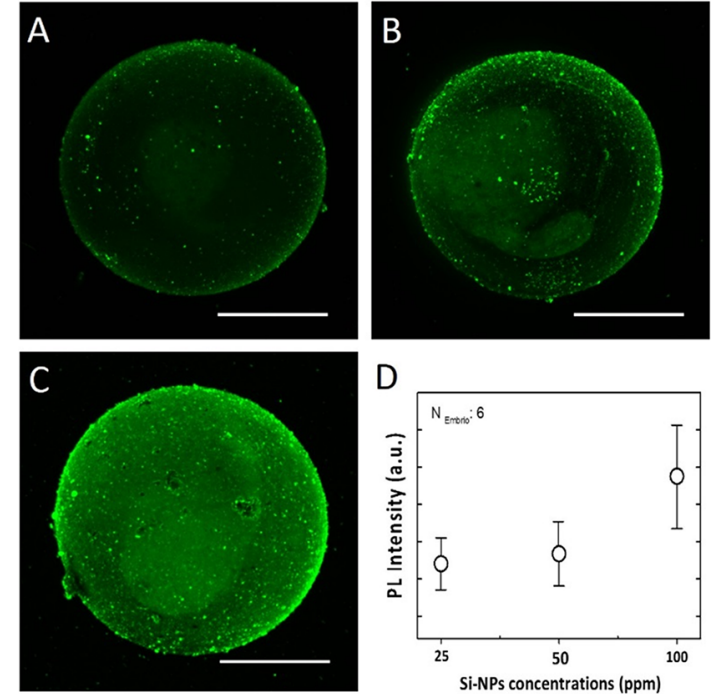

Figure 8. Maximum intensity projections of embryos at $24 \mathrm{hpf}$ treated with (A) 25, (B) 50, and (C) $100 \mathrm{ppm} \mathrm{Si-NPs.} \mathrm{Scale} \mathrm{bars} 500 \mu \mathrm{m}$. (D) Curve of Si-NPs fluorescence intensity vs concentration.

studies during the zebrafish development from 24 hpf until the completed developed larvae.

Si-NPs translocation inside zebrafish embryos and larvae was examined. Figure 9A shows the fluorescence intensity maximum projection of an embryo at $48 \mathrm{hpf}$ treated with $100 \mathrm{ppm}$ of Si-NPs, whereas Figure 9B-D represents three single z-planes with a distance of 200,400 , and $600 \mu \mathrm{m}$, respectively. We can observe that Si-NPs were successfully internalized (Figure 9B, white arrows), and mostly accumulated in embryos yolk sac (Figure 9C, white arrows). Although the zebrafish embryo (at $48 \mathrm{hpf}$ ) was still protected by the chorion, the presence of pore canals $(\approx 500 \mu \mathrm{m}$ in diameter $)$, allowed the $10 \mathrm{~nm}$ Si-NPs prepared by our protocol to passively enter in the embryo. ${ }^{69}$ On the contrary, others works reported that dye-functionalized silica NPs $(>50 \mathrm{~nm}$, green and red dye), i.e., no intrinsic NP emission, exposed to zebrafish absorbed on the surface of the embryos chorion without being taken up and translocated in the embryos. ${ }^{51}$

After the hatching at $72 \mathrm{hpf}$, in absence of chorion, the zebrafish larvae were able to internalize the Si-NPs by swallowing and absorption thorough the skin. ${ }^{69}$ Si-NPs were deposited in the yolk sac and gut and appear to be accumulated in the trunk of the larvae (Figure 10). Other works showed that polymeric grafted Si-NPs were mainly observed in the yolk sac. ${ }^{66}$ Moreover, Si-NPs prepared by our protocol accumulated in the lens of the eye, suggesting that
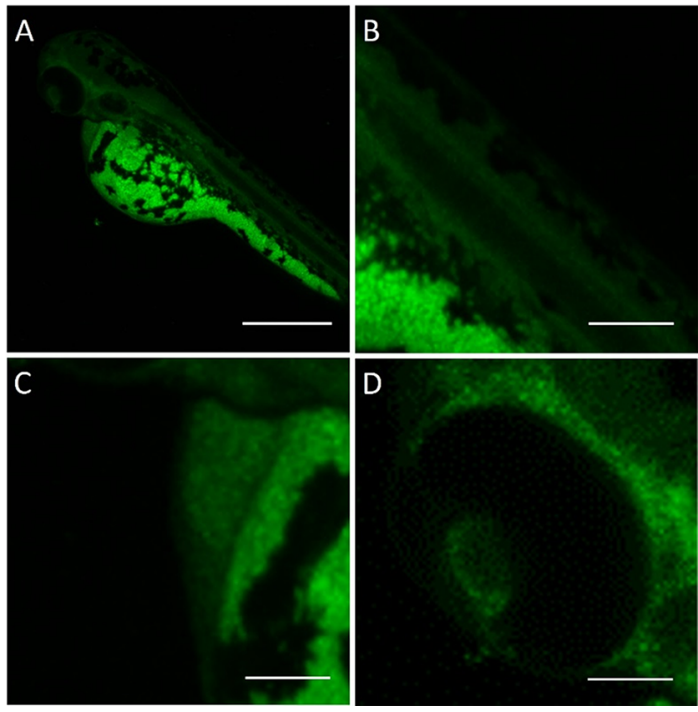

Figure 10. (A) Maximum intensity projection of the superior part of exposed larvae $(100 \mathrm{ppm})$ at $72 \mathrm{hpf}$. Scale bar $500 \mu \mathrm{m}$. (B) Zoom of the blood vessels, (C) zoom of the heart region, (D) zoom of the eye. Scale bars for $\mathrm{B}-\mathrm{D}=100 \mu \mathrm{m}$.

they enter in the eye crossing the blood ocular barrier (Figure 10D). Additionally, Si-NPs photoemission observed in the blood vessels and in the heart (Figure 10B, C) clearly demonstrated that Si-NPs entered in the circulatory system (see Video S1), and by this way they could reach different tissues and organs of the zebrafish. Zebrafish treated with only embryo medium as a control showed no significate fluorescence at the three different stages of growth investigated (data not shown).

\section{CONCLUSION}

In conclusion, we have reported on the fabrication and scaleup protocol of fluorescent, ligand-free Si-NPs (individual Si nanoparticles, $\sim 10 \mathrm{~nm}$ ) via ultraviolet liquid-phase pulsed laser ablation and their 3D biodistribution imaging and adverse effects studies, during early development zebrafish embryos. We showed that the Si-NPs hydrodynamic size was controlled by the adjustment of laser irradiation time. Post-treatment process demonstrated the feasibility to obtain high yield, i.e., $\sim 72 \%$, and monodisperse $10 \mathrm{~nm} \mathrm{Si-NPs} \mathrm{exhibiting} \mathrm{photo-}$ emission in the blue-green spectral range. Furthermore, we reported the nontoxicity, biosafety, and 3D imaging of ligandfree $\mathrm{Si}-\mathrm{NPs}$ in zebrafish embryos and larvae by assessing multiple biological parameters: no toxicity of ligand-free SiNPs was found at the different developmental stages even at
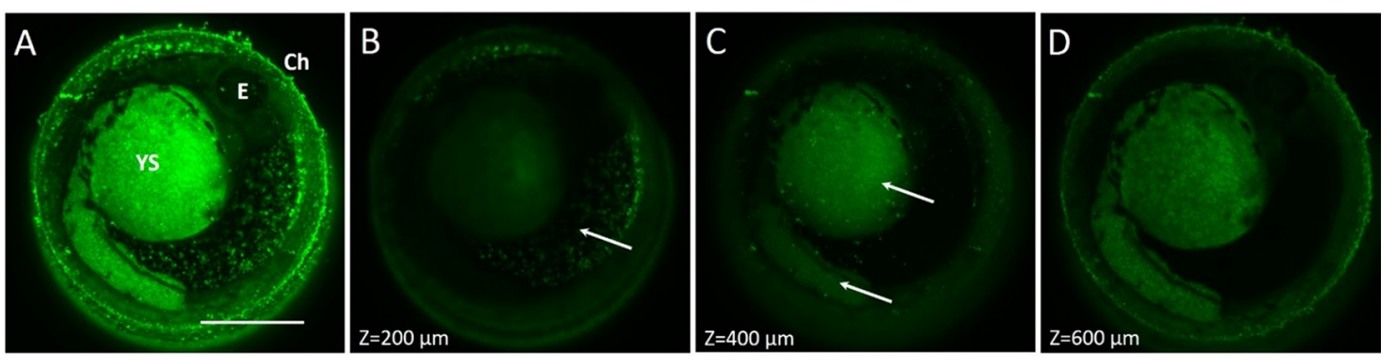

Figure 9. (A) Maximum intensity projections of $48 \mathrm{hpf}$ embryos treated with $100 \mathrm{ppm}$ Si-NPs. Scale bar $500 \mu \mathrm{m}$. (B-D) Images at different depths (step size $=200 \mu \mathrm{m})$. YS, yolk sac; E, eye; Ch, chorion. 
the higher concentration evaluated $\left(100 \mu \mathrm{g} \mathrm{mL}{ }^{-1}\right)$. The 3D imaging capability analysis permitted us to observe that the 10 $\mathrm{nm}$ Si-NPs can passively enter inside embryos by pore canals on the chorion, can diffuse in the circulatory system, i.e., blood vessel, and mostly accumulated in the zebrafish yolk sac and gut. Additionally, the accumulation of Si-NPs in the lens of the eye indicated that they were able to enter in the eye, crossing the blood ocular barrier. Our results evidently reported that ligand-free Si-NPs $(\sim 10 \mathrm{~nm})$ possess high biosafety making these nanoparticles appropriate for nanomedicine applications and are promising as a versatile biocompatible nanotools for fluorescence imaging and probing of biological systems.

\section{ASSOCIATED CONTENT}

\section{S Supporting Information}

The Supporting Information is available free of charge on the ACS Publications website at DOI: 10.1021/acsabm.8b00609.

DLS analysis of $\mathrm{Si}$ colloidal solution before and after treatment; absorption spectra of Si-NPs colloidal solution and $\mathrm{Si}$ concentration evaluated by ICP-EOS in the supernatant solution and in the pellet solution after treatment; HR-TEM and TEM observation of posttreated $\mathrm{Si}-\mathrm{NPs}$ in different grid areas; confocal microscopy emission spectrum of Si-NPs dispersed in water at $50 \mu \mathrm{g} \mathrm{mL}^{-1}$; FTIR and XPS analysis of the SiNPs colloidal solution after post treatment; optical images, heartbeat rate, and locomotor activity of zebrafish larvae treated with $\mathrm{Si}-\mathrm{NPs}$ (PDF) Video S1, zebra treated with Si-NPs (AVI)

\section{AUTHOR INFORMATION}

\section{Corresponding Author}

*Email: romuald.intartaglia@iit.it.

\section{ORCID $\odot$}

Romuald Intartaglia: 0000-0003-0415-1431

\section{Author Contributions}

${ }^{\dagger}$ M.d.A and M.R. contributed equally to this work.

\section{Author Contributions}

M.d.A, M.R., and R.I. performed the biological, synthesis, and characterization experiments and wrote the manuscript. G.S. and M.d.A. performed imaging experiments. The manuscript was revised and improved by all authors. All authors approved the final manuscript.

\section{Notes}

The authors declare no competing financial interest.

\section{ACKNOWLEDGMENTS}

We thank Dr. Monica Dalmonte for helping with the imaging measurements and acknowledge the Fondazione Istituto Italiano di Tecnologia and NIC@IIT center.

\section{REFERENCES}

(1) Murthy, S. K. Nanoparticles in Modern Medicine: State of the Art and Future Challenges. Int. J. Nanomed. 2007, 2 (2), 129-141.

(2) Yu, M.; Lin, Y.; Liu, Y.; Zhou, Y.; Liu, C.; Dong, L.; Cheng, K.; Weng, W.-J.; Wang, H. Enhanced Osteointegration of Hierarchical Structured 3D-Printed Titanium Implants. ACS Appl. Bio Mater. 2018, 1 (1), 90-99.

(3) He, X.; Shen, X.; Li, D.; Liu, Y.; Jia, K.; Liu, X. Dual-Mode Fluorescence and Magnetic Resonance Imaging Nanoprobe Based on Aromatic Amphiphilic Copolymer Encapsulated CdSe@ CdS and Fe3O4. ACS Appl. Bio Mater. 2018, 1 (2), 520-528.
(4) Grüner, M. C.; Arai, M.; Carreira, M.; Inada, N.; de Camargo, A. S. S. Functionalizing the Mesoporous Silica Shell of Upconversion Nanoparticles to Enhance Bacterial Targeting and Killing via Photosensitizer Induced aPDT. ACS Appl. Bio Mater. 2018, 1 (4), $1028-1036$.

(5) Wu, F.; Su, H.; Cai, Y.; Wong, W.-K.; Jiang, W.; Zhu, X. Porphyrin-implanted Carbon Nanodots for Photoacoustic Imaging and in Vivo Breast Cancer Ablation. ACS Appl. Bio Mater. 2018, 1 (1), 110-117.

(6) Maji, S. K.; Sreejith, S.; Joseph, J.; Lin, M.; He, T.; Tong, Y.; Sun, H.; Yu, S. W.-K.; Zhao, Y. Upconversion Nanoparticles as a Contrast Agent for Photoacoustic Imaging in Live Mice. Adv. Mater. 2014, 26 (32), 5633-5638.

(7) Hashim, Z.; Green, M.; Chung, P. H.; Suhling, K.; Protti, A.; Phinikaridou, A.; Botnar, R.; Khanbeigi, R. A.; Thanou, M.; Dailey, L. A.; Commander, N. J.; Rowland, C.; Scott, J.; Jenner, D. GdContaining Conjugated Polymer Nanoparticles: Bimodal Nanoparticles for Fluorescence and MRI Imaging. Nanoscale 2014, 6 (14), 8376-8386.

(8) Ambrosone, A.; Mattera, L.; Marchesano, V.; Quarta, A.; Susha, A. S.; Tino, A.; Rogach, A. L.; Tortiglione, C. Mechanisms Underlying Toxicity Induced by CdTe Quantum Dots Determined in an Invertebrate Model Organism. Biomaterials 2012, 33 (7), 1991-2000.

(9) Bozich, J. S.; Lohse, S. E.; Torelli, M. D.; Murphy, C. J.; Hamers, R. J.; Klaper, R. D. Surface Chemistry, Charge and Ligand Type Impact the Toxicity of Gold Nanoparticles to Daphnia Magna. Environ. Sci.: Nano 2014, 1 (3), 260-270.

(10) d'Amora, M.; Rodio, M.; Diaspro, A.; Intartaglia, R. EcoFriendly Processing for Engineering Bio-Safe Quantum Dots and their Interaction with Biological Systems. Biophys. J. 2017, 112 (3), 26 a.

(11) Park, J. H.; Gu, L.; von Maltzahn, G.; Ruoslahti, E.; Bhatia, S. N.; Sailor, M. J. Biodegradable Luminescent Porous Silicon Nanoparticles for In Vivo Applications. Nat. Mater. 2009, 8 (4), 331-336.

(12) Croissant, J. G.; Fatieiev, Y.; Khashab, N. M. Degradability and Clearance of Silicon, Organosilica, Silsesquioxane, Silica Mixed Oxide, and Mesoporous Silica Nanoparticles. Adv. Mater. 2017, 29 (9), 1604634.

(13) Chinnathambi, S.; Chen, S.; Ganesan, S.; Hanagata, N. Silicon Quantum Dots for Biological Applications. Adv. Healthcare Mater. 2014, 3 (1), 10-29.

(14) Henstock, J. R.; Canham, L. T.; Anderson, S. I. Silicon: The Evolution of its Use in Biomaterials. Acta Biomater. 2015, 11, 17-26.

(15) Bagga, K.; Barchanski, A.; Intartaglia, R.; Dante, S.; Marotta, R.; Diaspro, A.; Sajti, C. L.; Brandi, F. Laser-Assisted Synthesis of Staphylococcus Aureus Protein-capped Silicon Quantum Dots as Biofunctional Nanoprobes. Laser Phys. Lett. 2013, 10 (6), No. 065603.

(16) Gongalsky, M.; Osminkina, L.; Pereira, A.; Manankov, A.; Fedorenko, A.; Vasiliev, A.; Solovyev, V.; Kudryavtsev, A.; Sentis, M.; Kabashin, A. Laser-Synthesized Oxide-Passivated Bright Si Quantum Dots for Bioimaging. Sci. Rep. 2016, 6, 24732.

(17) Erogbogbo, F.; Yong, K. T.; Roy, I.; Hu, R.; Law, W. C.; Zhao, W. W.; Ding, H.; Wu, F.; Kumar, R.; Swihart, M. T.; Prasad, P. N. In Vivo Targeted Cancer Imaging, Sentinel Lymph Node Mapping and Multi-Channel Imaging with Biocompatible Silicon Nanocrystals. ACS Nano 2011, 5 (1), 413-423.

(18) Klein, S.; Zolk, O.; Fromm, M. F.; Schrödl, F.; Neuhuber, W.; Kryschi, C. Functionalized Silicon Quantum dots Tailored for Targeted siRNA Delivery. Biochem. Biophys. Res. Commun. 2009, 387 (1), 164-168.

(19) Wang, Q.; Bao, Y.; Ahire, J.; Chao, Y. Co-encapsulation of Biodegradable Nanoparticles with Silicon Quantum Dots and Quercetin for Monitored Delivery. Adv. Healthcare Mater. 2013, 2 (3), 459-466.

(20) Wareing, N.; Szymanski, K.; Akkaraju, G. R.; Loni, A.; Canham, L. T.; Gonzalez-Rodriguez, R.; Coffer, J. L. In Vitro Gene Delivery with Large Porous Silicon Nanoparticles Fabricated Using CostEffective, Metal-Assisted Chemical Etching. Small 2017, 13 (3), 1602739 . 
(21) Tamarov, K. P.; Osminkina, L. A.; Zinovyev, S. V.; Maximova, K. A.; Kargina, J. V.; Gongalsky, M. B.; Ryabchikov, Y.; Al-Kattan, A.; Sviridov, A. P.; Sentis, M.; Ivanov, A. V.; Nikiforov, V. N.; Kabashin, A. V.; Timoshenko, V. Y. Radio Frequency Radiation-Induced Hyperthermia using Si Nanoparticle-Based Sensitizers for Mild Cancer Therapy. Sci. Rep. 2015, 4, 7034.

(22) Rioux, D.; Laferrière, M.; Douplik, A.; Shah, D.; Lilge, L.; Kabashin, A. V.; Meunier, M. M. Silicon Nanoparticles Produced by Femtosecond Laser Ablation in Water as Novel Contamination-Free Photosensitizers. J. Biomed. Opt. 2009, 14 (2), No. 021010.

(23) Zhong, Y.; Sun, X.; Wang, S.; Peng, F.; Bao, F.; Su, Y.; Li, Y.; Lee, S.-T.; He, Y. Facile, Large-Quantity Synthesis of Stable, TunableColor Silicon Nanoparticles and Their Application for Long-Term Cellular Imaging. ACS Nano 2015, 9 (6), 5958-5967.

(24) Wu, S.; Zhong, Y.; Zhou, Y.; Song, B.; Chu, B.; Ji, X.; Wu, Y.; $\mathrm{Su}, \mathrm{Y}$.; He, Y. Biomimetic Preparation and Dual-Color Bioimaging of Fluorescent Silicon Nanoparticles. J. Am. Chem. Soc. 2015, 137 (46), 14726-14732.

(25) Rodio, M.; Brescia, R.; Diaspro, A.; Intartaglia, R. Direct Surface Modification of Ligand-Free Silicon Quantum Dots Prepared by Femtosecond Laser Ablation in Deionized Water. J. Colloid Interface Sci. 2016, 465, 242-248.

(26) Körösi, L.; Rodio, M.; Dömötör, D.; Kovács, T.; Papp, S.; Diaspro, A.; Intartaglia, R.; Beke, S. Ultrasmall Ligand-Free Ag Nanoparticles with High Antibacterial Activity Prepared by Pulsed Laser Ablation in Liquid. J. Chem. 2016, 2016, 8.

(27) Rodio, M.; Scarpellini, A.; Diaspro, A.; Intartaglia, R. Tailoring of Size, Emission and Surface Chemistry of Germanium Nanoparticles via Liquid-Phase Picosecond Laser Ablation. J. Mater. Chem. C 2017, 5 (46), 12264-12271.

(28) García-Calzada, R.; Rodio, M.; Bagga, K.; Intartaglia, R.; Bianchini, P.; Chirvony, V. S.; Martínez-Pastor, J. P. Facile LaserAssisted Synthesis of Inorganic Nanoparticles Covered by a Carbon Shell with Tunable Luminescence. RSC Adv. 2015, 5 (62), 5060450610 .

(29) Intartaglia, R.; Barchanski, A.; Bagga, K.; Genovese, A.; Das, G.; Wagener, P.; Di Fabrizio, E.; Diaspro, A.; Brandi, F.; Barcikowski, S. Bioconjugated Silicon Quantum Dots from One-Step Green Synthesis. Nanoscale 2012, 4 (4), 1271-1274.

(30) Rodio, M.; Coluccino, L.; Romeo, E.; Genovese, A.; Diaspro, A.; Garau, G.; Intartaglia, R. Facile Fabrication of Bioactive UltraSmall Protein-Hydroxyapatite Nanoconjugates via Liquid-Phase Laser Ablation and their Enhanced Osteogenic Differentiation Activity. $J$ Mater. Chem. B 2017, 5 (2), 279-288.

(31) Amendola, V.; Meneghetti, M. What controls the Composition and the Structure of Nanomaterials generated by Laser Ablation in Liquid Solution? Phys. Chem. Chem. Phys. 2013, 15 (9), 3027-3046.

(32) Blandin, P.; Maximova, K. A.; Gongalsky, M. B.; Sanchez-Royo, J. F.; Chirvony, V. S.; Sentis, M.; Timoshenko, V. Y.; Kabashin, A. V. Femtosecond Laser Fragmentation from Water-Dispersed Microcolloids: Toward Fast Controllable Growth of Ultrapure Si-Based Nanomaterials for Biological Applications. J. Mater. Chem. B 2013, 1 (19), 2489-2495.

(33) Baati, T.; Al-Kattan, A.; Esteve, M.-A.; Njim, L.; Ryabchikov, Y.; Chaspoul, F.; Hammami, M.; Sentis, M.; Kabashin, A. V.; Braguer, D. Ultrapure Laser-Synthesized Si-based Nanomaterials for Biomedical Applications: In Vivo Assessment of Safety and Biodistribution. Sci. Rep. 2016, 6, 25400.

(34) Intartaglia, R.; Bagga, K.; Brandi, F.; Das, G.; Genovese, A.; Di Fabrizio, E.; Diaspro, A. Optical Properties of Femtosecond LaserSynthesized Silicon Nanoparticles in Deionized Water. J. Phys. Chem. C 2011, 115 (12), 5102-5107.

(35) Hamad, S.; Podagatlapalli, G. K.; Vendamani, V.; Nageswara Rao, S.; Pathak, A.; Tewari, S. P.; Venugopal Rao, S. Femtosecond Ablation of Silicon in Acetone: Tunable Photoluminescence from Generated Nanoparticles and Fabrication of Surface Nanostructures. J. Phys. Chem. C 2014, 118 (13), 7139-7151.

(36) Intartaglia, R.; Bagga, K.; Brandi, F. Study on the Productivity of Silicon Nanoparticles by Picosecond Laser Ablation in Water:
Towards Gram Per Hour Yield. Opt. Express 2014, 22 (3), 31173127.

(37) Zhang, Y.; Wu, W.; Hao, H.; Shen, W. Femtosecond LaserInduced Size Reduction and Emission Quantum Yield Enhancement of Colloidal Silicon Nanocrystals: Effect of Laser Ablation Time. Nanotechnology 2018, 29 (36), 365706.

(38) Chewchinda, P.; Tsuge, T.; Funakubo, H.; Odawara, O.; Wada, H. Laser Wavelength Effect on Size and Morphology of Silicon Nanoparticles Prepared by Laser Ablation in Liquid. Jpn. J. Appl. Phys. 2013, 52 (2R), No. 025001.

(39) Kuzmin, P. G.; Shafeev, G. A.; Bukin, V. V.; Garnov, S. V.; Farcau, C.; Carles, R.; Warot-Fontrose, B.; Guieu, V.; Viau, G. Silicon Nanoparticles Produced by Femtosecond Laser Ablation in Ethanol: Size Control, Structural Characterization, and Optical Properties. J. Phys. Chem. C 2010, 114 (36), 15266-15273.

(40) Alkis, S.; Okyay, A. K.; Ortaç, B. Post-Treatment of Silicon Nanocrystals Produced by Ultra-Short Pulsed Laser Ablation in Liquid: Toward Blue Luminescent Nanocrystal Generation. J. Phys. Chem. C 2012, 116 (5), 3432-3436.

(41) Napierska, D.; Thomassen, L. C.; Lison, D.; Martens, J. A.; Hoet, P. H. The Nanosilica Hazard: another Variable Entity. Part. Fibre Toxicol. 2010, 7 (1), 39.

(42) Wang, Q.; Bao, Y.; Zhang, X.; Coxon, P. R.; Jayasooriya, U. A.; Chao, Y. Uptake and Toxicity Studies of Poly-Acrylic Acid Functionalized Silicon Nanoparticles in Cultured Mammalian Cells. Adv. Healthcare Mater. 2012, 1 (2), 189-198.

(43) Tolstik, E.; Osminkina, L. A.; Matthaus, C.; Burkhardt, M.; Tsurikov, K. E.; Natashina, U. A.; Timoshenko, V. Y.; Heintzmann, R.; Popp, J.; Sivakov, V. Studies of Silicon Nanoparticles Uptake and Biodegradation in Cancer Cells by Raman Spectroscopy. Nanomedicine (N. Y., NY, U. S.) 2016, 12 (7), 1931-1940.

(44) Liu, J.; Erogbogbo, F.; Yong, K.-T.; Ye, L.; Liu, J.; Hu, R.; Chen, H.; Hu, Y.; Yang, Y.; Yang, J.; Roy, I.; Karker, N. A.; Swihart, M. T.; Prasad, P. N. Assessing Clinical Prospects of Silicon Quantum Dots: Studies in Mice and Monkeys. ACS Nano 2013, 7 (8), 73037310.

(45) Bimbo, L. M.; Sarparanta, M.; Santos, H. A.; Airaksinen, A. J.; Mäkilä, E.; Laaksonen, T.; Peltonen, L.; Lehto, V.-P.; Hirvonen, J.; Salonen, J. Biocompatibility of Thermally Hydrocarbonized Porous Silicon Nanoparticles and their Biodistribution in Rats. ACS Nano 2010, 4 (6), 3023-3032.

(46) d'Amora, M.; Sancataldo, G.; Intartaglia, R.; Diaspro, A. In Vivo Nano-Bio Interactions in Zebrafish using Advanced Fluorescence Microscopy. Biophys. J. 2017, 112 (3), 303a.

(47) Rizzo, L. Y.; Golombek, S. K.; Mertens, M. E.; Pan, Y.; Laaf, D.; Broda, J.; Jayapaul, J.; Möckel, D.; Subr, V.; Hennink, W. E.; et al. In Vivo Nanotoxicity Testing using the Zebrafish Embryo Assay. J. Mater. Chem. B 2013, 1 (32), 3918-3925.

(48) d'Amora, M.; Camisasca, A.; Lettieri, S.; Giordani, S. Toxicity Assessment of Carbon Nanomaterials in Zebrafish during Development. Nanomaterials 2017, 7 (12), 414.

(49) Busquet, F.; Nagel, R.; von Landenberg, F.; Mueller, S. O.; Huebler, N.; Broschard, T. H. Development of a new Screening Assay to identify Proteratogenic Substances using Zebrafish Danio rerio Embryo combined with an Exogenous Mammalian Metabolic Activation System (mDarT). Toxicol. Sci. 2008, 104 (1), 177-188.

(50) Duan, J.; Yu, Y.; Shi, H.; Tian, L.; Guo, C.; Huang, P.; Zhou, X.; Peng, S.; Sun, Z. Toxic Effects of Silica Nanoparticles on Zebrafish Embryos and Larvae. PLoS One 2013, 8 (9), No. e74606.

(51) Fent, K.; Weisbrod, C. J.; Wirth-Heller, A.; Pieles, U. Assessment of Uptake and Toxicity of Fluorescent Silica Nanoparticles in Zebrafish (Danio rerio) Early Life Stages. Aquat. Toxicol. 2010, 100 (2), 218-228.

(52) d' Amora, M.; Rodio, M.; Bartelmess, J.; Sancataldo, G.; Brescia, R.; Cella Zanacchi, F.; Diaspro, A.; Giordani, S. Biocompatibility and Biodistribution of Functionalized Carbon Nano-onions (f-CNOs) in a Vertebrate Model. Sci. Rep. 2016, 6, 33923. 
(53) Shilo, M.; Sharon, A.; Baranes, K.; Motiei, M.; Lellouche, J.-P. M.; Popovtzer, R. The Effect of Nanoparticle Size on the Probability to Cross the Blood-Brain Barrier: an In-Vitro Endothelial Cell Model. J. Nanobiotechnol. 2015, 13, 19.

(54) Manhat, B. A.; Brown, A. L.; Black, L. A.; Ross, J. B. A.; Fichter, K.; Vu, T.; Richman, E.; Goforth, A. M. One-Step Melt Synthesis of Water-Soluble, Photoluminescent, Surface-Oxidized Silicon Nanoparticles for Cellular Imaging Applications. Chem. Mater. 2011, 23 (9), 2407-2418.

(55) Intartaglia, R.; Bagga, K.; Scotto, M.; Diaspro, A.; Brandi, F. Luminescent Silicon Nanoparticles Prepared by Ultra Short Pulsed Laser Ablation in Liquid for Imaging Applications. Opt. Mater. Express 2012, 2 (5), 510-518.

(56) Fitting, H.-J.; Barfels, T.; Trukhin, A.; Schmidt, B.; Gulans, A.; Von Czarnowski, A. Cathodoluminescence of $\mathrm{Ge}+, \mathrm{Si}+$, and $\mathrm{O}+$ Implanted $\mathrm{SiO} 2$ Layers and the Role of Mobile Oxygen in Defect Transformations. J. Non-Cryst. Solids 2002, 303 (2), 218-231.

(57) Pi, X. D.; Liptak, R. W.; Nowak, J. D.; Wells, N. P.; Carter, C. B.; Campbell, S. A.; Kortshagen, U. Air-Stable Full-Visible-Spectrum Emission from Silicon Nanocrystals Synthesized by an All-Gas-Phase Plasma Approach. Nanotechnology 2008, 19 (24), 245603.

(58) Tan, D.; Ma, Z.; Xu, B.; Dai, Y.; Ma, G.; He, M.; Jin, Z.; Qiu, J. Surface Passivated Silicon Nanocrystals with Stable Luminescence Synthesized by Femtosecond Laser Ablation in Solution. Phys. Chem. Chem. Phys. 2011, 13 (45), 20255-20261.

(59) Llansola Portolés, M. J.; Rodriguez Nieto, F.; Soria, D. B.; Amalvy, J. I.; Peruzzo, P. J.; Mártire, D. O.; Kotler, M.; Holub, O.; Gonzalez, M. C. Photophysical Properties of Blue-Emitting Silicon Nanoparticles. J. Phys. Chem. C 2009, 113 (31), 13694-13702.

(60) Llansola Portolés, M. J.; Rodriguez Nieto, F.; Soria, D. B.; Amalvy, J. I.; Peruzzo, P. J.; Mártire, D. O.; Kotler, M.; Holub, O.; Gonzalez, M. C. Photophysical Properties of Blue-Emitting Silicon Nanoparticles. J. Phys. Chem. C 2009, 113 (31), 13694-13702.

(61) Yang, D. Q.; Ethier, V.; Sacher, E.; Meunier, M. Photoluminescence of Highly Porous Nanostructured Si-based Thin Films Deposited by Pulsed Laser Ablation. J. Appl. Phys. 2005, 98 (2), No. 024310.

(62) Brunet-Bruneau, A.; Souche, D.; Fisson, S.; Van, V. N.; Vuye, G.; Abeles, F.; Rivory, J. Microstructural Characterization of Ion Assisted $\mathrm{SiO} 2$ Thin Films by Visible and Infrared Ellipsometry. J. Vac. Sci. Technol., A 1998, 16 (4), 2281-2286.

(63) Xu, D.; Sun, L.; Li, H.; Zhang, L.; Guo, G.; Zhao, X.; Gui, L. Hydrolysis and Silanization of the Hydrosilicon Surface of Freshly Prepared Porous Silicon by an Amine Catalytic Reaction. New J. Chem. 2003, 27 (2), 300-306.

(64) Gupta, P.; Dillon, A. C.; Bracker, A. S.; George, S. M. FTIR Studies of $\mathrm{H} 2 \mathrm{O}$ and D2O Decomposition on Porous Silicon Surfaces. Surf. Sci. 1991, 245 (3), 360-372.

(65) Li, Z. F.; Swihart, M. T.; Ruckenstein, E. Luminescent Silicon Nanoparticles Capped by Conductive Polyaniline through the SelfAssembly Method. Langmuir 2004, 20 (5), 1963-1971.

(66) Fan, J.-W.; Vankayala, R.; Chang, C.-L.; Chang, C.-H.; Chiang, C.-S.; Hwang, K. C. Preparation, Cytotoxicity and In Vivo Bioimaging of Highly Luminescent Water-Soluble Silicon Quantum Dots. Nanotechnology 2015, 26 (21), 215703.

(67) Chen, T. H.; Lin, C. Y.; Tseng, M. C. Behavioral Effects of Titanium Dioxide Nanoparticles on Larval Zebrafish (Danio rerio). Mar. Pollut. Bull. 2011, 63, 303-308.

(68) Duan, J.; Yu, Y.; Li, Y.; Yu, Y.; Li, Y.; Huang, P.; Zhou, X.; Peng, S.; Sun, Z. Developmental Toxicity of CdTe QDs in Zebrafish Embryos and Larvae. J. Nanopart. Res. 2013, 15 (7), 1700.

(69) Lee, K. J.; Nallathamby, P. D.; Browning, L. M.; Osgood, C. J.; $\mathrm{Xu}, \mathrm{X} . \mathrm{-H}$. N. In Vivo Imaging of Transport and Biocompatibility of Single Silver Nanoparticles in Early Development of Zebrafish Embryos. ACS Nano 2007, 1 (2), 133-143. 